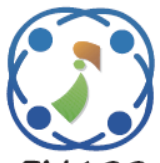

\title{
Probabilistic Optimal Power Dispatch Considering Price-Based Real-Time Demand Response
}

\author{
Udoum Chhor $^{1} \quad$ Uthen Leeton $^{1} \quad$ Keerati Chayakulkheeree $^{1 *}$ \\ ${ }^{I}$ School of Electrical Engineering, Institute of Engineering, Suranaree University of Technology, Thailand \\ * Corresponding author's Email: keerati.ch@sut.ac.th
}

\begin{abstract}
In this paper, a probabilistic optimal power dispatch (POPD) using linear programming (LP) is proposed for solving the power generation dispatch with price-based real-time demand response (PRDR). The expected shortterm load forecast is represented by a probabilistic distribution function. The simulation result has prosperously shown that the proposed method could handle the POPD solutions for real power dispatch considering PRDR by using probabilistic Truncated normal distribution function (PTNF). The PTNF is used in a vigorous part of the framework to eject the infeasible results during the computation. This would lead the results to the high degree of accuracy comparing to the normal sampling methods. The simulation results showed that the proposed method can efficiently and effectively minimize the total power generation cost while trading off the PRDR cost in the POPD problem with load uncertainty.
\end{abstract}

Keywords: Probabilistic optimal power dispatch, Probability density function, Linear programming, Demand response.

\section{Introduction}

In the power grid, the electric utilities need to balance the power generation and load considering economic operation considering grid reliability and quality of supply. Therefore, optimal power dispatch (OPD) techniques for possible future power system operation are steadily proposed with various optimization techniques.

Recently, the innovation of computer processor units has produced as a matter of engineering required to solve their problems as fast as possible in real time and online. OPD has become one of the most extensive optimization tools adopted in the power system planning and electricity market. With the above issues, many researchers have endlessly studied optimization techniques to investigate an optimum operation of the power system. Many optimization algorithms have always been mentioned both artificial intelligence and conventional methods to obtain an OPD solution. The linear programming (LP) is one of the most conventional methods which becomes a widely practical method in optimal power system operation. For example, a demand response (DR) strategy based on energy consumption scheduling was modelled by LP to prove the demand minimizing in peak period in [1]. The marker prices are exposed by LP proposed framework equivalent to the marginal cost for the utility in [2]. Similarly, it was used to minimalize the expensive fuel operating cost in extra high voltage in [3]. Another LP proposed algorithm is to minimize the supply cost in power pool auction. In the power pool auction, the hourly bus spot price incorporating the marginal transmission loss and network quality of supply can be regulated [4-5]. LP has the potential to capture optimal adaptive operating costs and provide the optimal dispatch module in both short and long terms optimization problems, such as numerous economic, social, military and real-time problems. In practice, the short-term load forecast for hourahead dispatch is usually uncertain in nature. Therefore, the probabilistic model representation for the system loading can be used to deal with uncertainty. 
In trendy power grid, DR programs have been developed and studied in many researches in modern power systems. The purpose of developing DR models is to provide accurate dispatch balance and stability analysis of future grid. DR is a specific program to motivate the end users' response to reduce or rearrange the electricity usage patterns during critical peak time. In developing an approach of the modern power grid, some models of DR have implemented to manage the higher prices during the peak demand in the system to avoid increasing power generation. Meanwhile, consumers have always billed their energy consumption through a tariff depending on the users' demands and had no any economic instructions or reports on how to plan to use or shift the consumption during peak periods. The aims of the evaluation methodology are to prove the peak demand and power consumption in economizing the total operating cost efficiency associated with DR program are extracted in [6]. Real-time Pricing (RTP) is a well-known prospect of DR scheme proposed by the system operator (SO) [7-8]. Aggregated consumers are encouraged to draw attention to reduce their demands accordingly to the required power balance in the system reliability. The DR programs in which price variations of energy over time produce changes at consumers' demand profile. It is necessary to improve the above problems to balance between supply and power demand side. To sum up, there are more details on DR programming and optimization algorithms [9], practical indication and key-elements for global experience [10], demand-side elasticity and DR budding [11], bearing investigation with its solution [12], and uncertainties in power systems [13].

In order to investigate the output target of the power system, there are three broadly used methods to solve the POPD problems such as analytical, approximation, and simulation methods. One of the most powerful techniques for POPD is Monte Carlo simulation (MCS) which is extensively used method to deal with uncertainties in the power system; it is relied on repeated random sampling to get the numerical results and reliability analysis statistically. In the proposed framework, the normal probability density function (PDF) was transformed to be the Truncated normal PDF, and it was shown that small errors occurred in the computed expected values which could be compensated for by shifting the computed probability-density curve so that its expected value coincided with the value deduced from a conventional deterministic analysis. It was formerly used to examine how probabilistic load flow (PLF) can be evaluated and found out the greater accuracy throughout the computational optimum speed [14]. Another point of view, MCS is used to perform the probabilistic short-term load forecast scheduling in a power system by assuming the PDF as the system loading, the total operating cost is effectually optimized [15]. Furthermore, many similar researches have studied the effect of correlation of uncertain variables such as probabilistic appraisal of accessible load supply capability [16], POPF behavior and relationship of the wind power, load uncertainties and line parameters [17, 18], PLF for solar power using percentile estimation of Weibull PDF [19], probabilistic investigation when wind and photovoltaic generation connected to system [20] PLF based on correlated series of generation, loading, and wind farm [21], probabilistic comparison and evaluation with energy management application [22], economic dispatch relied on QuasiMCS is used to models the stochastic behaviors of wind speed and distributed loads [23], uncertainty of loads and wind speed is characterized by MCS to represent the total number of hours with overvoltage a year [24], hybrid MCS is performed to evaluate PLF when a large-scale wind power integrated to power system [25]. All these probabilistic problems and some other relevance are modelled in different purposes to balance the system loading by adjusting the add-on power generation in the power system.

In this paper, the linear programming optimal power dispatch (LPOPD) considering price-based real-time demand response (PRDR) is implemented in the modified IEEE 30-bus test system. Based on the problem formulation, the piecewise linear cost function is used to represent the generator's operating cost. At the same time, the PRDRs participate in dispatching aggregator loads connected to the system. The purpose is to accomplish the supply-demand balancing without upward power supply. Many works were developed in the smart grid, distributed generation, and other energy sources to serve the growing demands. Those additional generations will add the extra production cost and many complexities along. The simulation output of LPOPD with and without PRDR are addressed and compared in the results.

The proposed method accentuates the probabilistic inquiries in POPD solutions. The empirical rule will perform as a vital role in the computational procedure to avoid the infeasible load flow (LF) results during the computation to warrant the real-time simulation over the existing works is used the normal PDF to represent the uncertainty of variables in the system. The results will release preciously from simulation method time frame. 
Besides the introduction, the paper is consisted of: Section 2 introduces the model of uncertainties including DR schemes, expresses load modelling and probabilistic loading pattern. Section 3 represents the problem formulation of the POPD using LP with DR programs, while the real power demand at load bus is represented by normal PDF. Section 4 explains the probabilistic technique and conditions for sampling the input variables to represent the real power demand and rules for Truncated normal PDF to state a specific range for the random variable to obtain a better accuracy. Also, the proposed framework of Monte Carlo technique and performance of the simulation are denoted in this section. Section 5 indicates the simulation results from the modified 30-bus test system. Lastly, Section 6 provides the conclusion.

\section{DR schemes and probabilistic load models}

\subsection{DR schemes}

DR programs have essentially empowered because the evolution in the up-to-date technology required to tool them to regulate the target. An implication of DR is to consider the possibility of the power generation cost reduction, customers' electricity bill saving, and reliability of the power grid. PRDR is a program in which customers are paid for the load reduction in accordance to SO request. The PRDR price can be assigned by agreements for the real-time curtailable load. The demand of each load bus in the system has adjusted to maintain with the feasible power generation, principally, every customer would manage their power consumption to be a part of improving the efficiency and reliability of the system during peak periods. The system operator sometimes has to run costly power plant to adjust the total needs power generation to meet the peak demand while the promise pollution can be exceeded their authority, however, whether DR scheme has contributed to the system. Hence, there are persuasively two DR programs in vogue $[9,10]$ which are price-based programs (PBPs) and incentive-based programs (IBPs). PBPs are commonly cased study for researchers which provoke the consumers voluntarily provide load reductions by reacting to economic gestures. In spite of IBPs the customers have bided the payments in order to report an exact amount of load reduction over a specified time interval. Many economists are convinced that they are the most direct and efficient DR programs suitable for competitive electricity markets and should be the focus of policymakers.

\subsection{Load modelling}

In probability and statistics manner, random variables or stochastic variables are variables which represent possible numbers by using probability theories. Practically, the normal PDF is a common continuous probability distribution to produce realvalued random variables as load uncertainty. In this paper, the normal distributed random variable is used to model the real power demand on every bus.

For this purpose, the equivalent PDF can be formulated as,

$$
f\left(P_{D_{i}} \mid \mu_{D}, \sigma_{D}^{2}\right)=\frac{1}{\sigma_{D} \sqrt{2 \pi}} e^{-\frac{1}{2}\left(\frac{P_{D_{i}}-\mu_{D}}{\sigma_{D}}\right)^{2}},
$$

Where,

$P_{D_{i}} \quad$ is the probabilistic real power demand at bus $i$,

$\mu_{D} \quad$ is the mean value, and

$\sigma_{D} \quad$ is the standard deviation of the demand profile.

\subsection{Probabilistic and practical loading pattern}

For this simulation, a practical loading pattern of Thai power system [15] was selected to use as power demand data pattern by transforming into the normal PDF. The annual system loading at 14:00 was formed into normalized data and used in this paper.

Consistently, the normalized annual data has plotted a histogram of 365-day data while the normal PDF fit is explored in coordination with normal PDF curve as shown in Fig. 1. After that, the

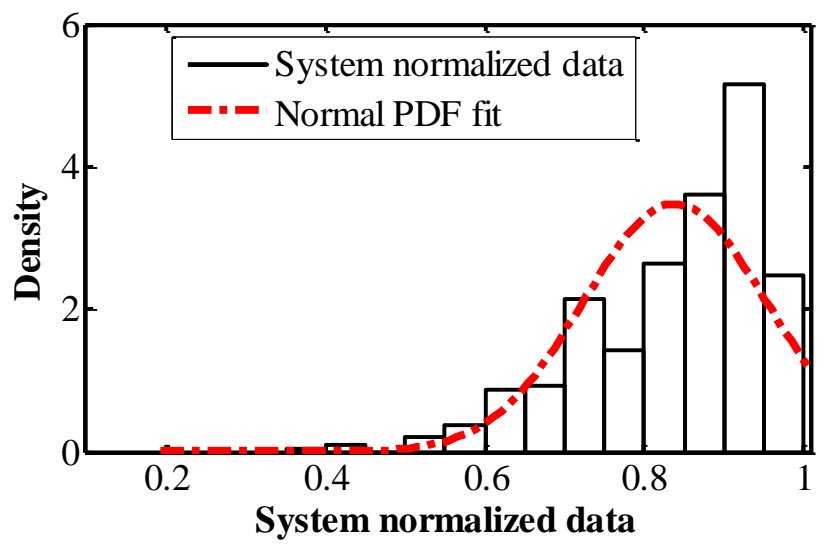

Figure. 1 PDF fitness for daily system loading at 14:00 
parameters $\mu_{D}$ and $\sigma_{D}$ will be obtained for using in the sampling conditions in section 4 .

\section{Problem formulation}

The objective of this paper is to minimize the total system investment cost in considering PRDR. The linear programming optimal power dispatch (LPOPD) is adapted to coordinate with NewtonRaphson power flow (NRPF). The power flow is used to obtain the losses and to test the feasibility of the dispatch solution.

\subsection{OPD modelling}

In this case study, the LPOPD adapted the NRPF with the operating cost for each generator which is given by piecewise linear cost functions. It can be used instead of the quadratic cost functions. Hence, the objective function can be expressed by a piecewise linear optimization model [4-5,15]. It is to minimize the total power generating cost including cost of PRDR, and can be expressed as,

Minimize $T C=\sum_{i=1}^{N G} \sum_{j=1}^{N S i} S_{i j} P_{G_{i j}}+\sum_{i=1}^{N B} D_{i} P_{D R_{i}}$,

subjected to the power balance constraint,

$$
\begin{aligned}
& P_{G_{i}}-P_{D_{i}}=\sum_{i=1}^{N B}\left|V_{i}\right|\left|V_{k}\right|\left|y_{i k}\right| \cos \left(\theta_{i k}-\delta_{i k}\right), i=1,2, \ldots, N B, \\
& Q_{G_{i}}-Q_{D_{i}}=-\sum_{i=1}^{N B}\left|V_{i}\right|\left|V_{k}\right|\left|y_{i k}\right| \sin \left(\theta_{i k}-\delta_{i k}\right), i=1,2, \ldots, N B, \\
& \sum_{i=1}^{N G} P_{G_{i}}+\sum_{i=1}^{N B} P_{D R_{i}}=\sum_{i=1}^{N B} P_{D_{i}}^{o}+P_{\text {loss }},
\end{aligned}
$$

and the generator operating limit constraint,

$$
\begin{aligned}
& P_{G_{i}}^{\min } \leq P_{G_{i}} \leq P_{G_{i}}^{\max }, i=1, \ldots, N G, \\
& \sum_{i=1}^{N G} P_{G_{i}}=\sum_{i=1}^{N B} P_{D_{i}}+P_{\text {loss }}, \\
& P_{D_{i}}=P_{D_{i}}^{o}-P_{D R_{i}}, i=1, \ldots, N B, \\
& P_{G_{i}}=\sum_{j=1}^{N S i} P_{G_{i j}}+P_{G_{i}}^{\min }, i=1, \ldots, N G, \\
& 0 \leq P_{G_{i j}} \leq P_{G_{i j}}^{\max }, j=1, \ldots, N S_{i}, \\
& \left|f_{l m}\right| \leq\left|f_{l m}\right|^{\max }, \\
& \left|V_{i}\right|^{\min } \leq\left|V_{i}\right|^{\leq}\left|V_{i}\right|^{\max }, i=1, \ldots, N B .
\end{aligned}
$$

Where,
TC is the total system cost,

$P_{G_{i}} \quad$ is the real power generation at bus $i$,

$S_{i j} \quad$ is the linearized incremental cost curve for each segment of $P_{G i}$ at bus $i$,

$D_{i} \quad$ is the linearized incremental cost curve for each demand response at bus $i$,

$N S_{i} \quad$ is the number of segments of the linearized cost of the generator at bus $i$,

$N G$ is the number of generators in the system,

$N B \quad$ is the number of buses in the system,

$P_{D R_{i}} \quad$ is the real power demand response at bus $i$,

$P_{D_{i}} \quad$ is the probabilistic real power demand at bus $i$,

$Q_{G_{i}} \quad$ is the reactive power generation at bus $i$,

$Q_{D_{i}} \quad$ is the reactive power demand at bus $i$,

$P_{\text {loss }}$ is the total transmission loss in the system,

$P_{G_{i}}^{\min } \quad$ is the minimum real power generation at bus $i$,

$P_{G_{i}}^{\max }$ is the maximum real power generation at bus $i$,

$\left|f_{l m}\right|$ is the apparent power flow on the branch between bus $l$ and $m$,

$\left|f_{l m}\right|^{\max }$ is the maximum limit at apparent power flow on the branch between bus $l$ and $m$,

$\left|V_{i}\right| \quad$ is the voltage magnitude at bus $i$,

$\left|V_{i}\right|^{\max }$ is the maximum voltage magnitude at bus $i$,

$\left|V_{i}\right|^{\min }$ is the minimum voltage magnitude at bus $i$,

$\left|y_{i k}\right|$ is the magnitude of the $y_{i k}$ element of $Y_{b u s}$,

$\theta_{i k} \quad$ is the angle of the $y_{i k}$ element of $Y_{b u s}$, and

$\delta_{i k} \quad$ is the voltage angle between bus $i$ and $k$.

\subsection{LPOPD algorithm}

The algorithm approach is based on an iterative computation between Newton-Raphson power flow (NRPF) and LP. The computational procedure is shown in Fig. 2.

\section{Probabilistic technique and sampling conditions}

\subsection{Conditional random variable}

Regarding section 2, the normal PDF is chosen to model the load uncertainty with the specified parameters $\mu_{D}$ and $\sigma_{D}$ obtained from the practical 


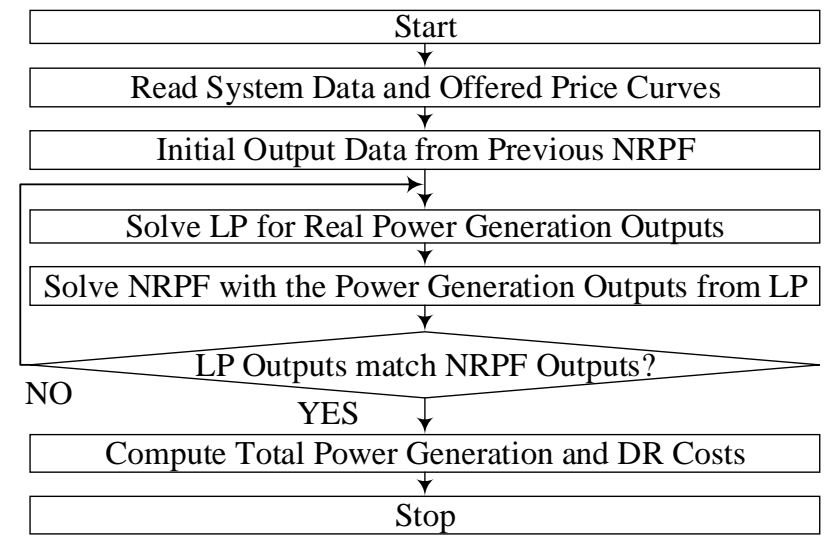

Figure. 2 Computational procedure of LPOPD

data as shown in Fig. 2. One of the most important aspects in this simulation is to execute a truncation range $x \in(a, b)$.

Suppose that $x \sim N\left(\mu_{D}, \sigma_{D}{ }^{2}\right),-\infty \leq a<b \leq \infty$. Then, the normal distribution has become the Truncated normal PDF lying on the interval $a<x<b$. In general, the Truncated normal PDF will be symbolized by $\Psi()$ [26]. And it is classified by the formula,

$\Psi\left(\mu_{D}, \sigma_{D}, a, b ; x\right)= \begin{cases}0 & \text { if } x \leq a \\ \Phi\left(\mu_{D}, \sigma_{D}{ }^{2}, a, b ; x\right) & \text { if } a<x<b \\ l & \text { if } b \leq x\end{cases}$

Where,

$\Phi\left(\mu_{D}, \sigma_{D}^{2}, a, b ; x\right)=\frac{\phi\left(\mu_{D}, \sigma_{D}^{2} ; x\right)-\phi\left(\mu_{D}, \sigma_{D}^{2} ; a\right)}{\phi\left(\mu_{D}, \sigma_{D}^{2} ; b\right)-\phi\left(\mu_{D}, \sigma_{D}^{2} ; a\right)}$

From the above summary, it is clearly shown that $\Psi()$ is 0 at $x \leq a, 1$ at $b \leq x$, and it is in-between the shifted version of the behaviour of $\Phi()$ at $a<x<$ $b$.

\subsection{Rules for truncated normal PDF data}

In statistics, there is a rule called the 68-9599.7 rule to deal around the mean value in the normal distribution, sometimes known as the empirical rule [13, 27-29], in order to get more accurately, $68.27 \%, 95.45 \%$ and $99.73 \%$ of the random variables within one standard deviation, two standard deviations, and three standard deviations of the mean, respectively.

To formulate the data in this study, the approximated normal PDF data set aimed at empirical data derivation. In this case, vector $\mathrm{x}$ generated randomly on a specific range, represented by $x_{(a, b)}=\left[x_{1}, x_{2}, \ldots, x_{m c s}\right]$ which samples depending on how many times MCS will simulate in the 68-9599.7 rule framework. The standard deviation $\sigma_{D}$ of

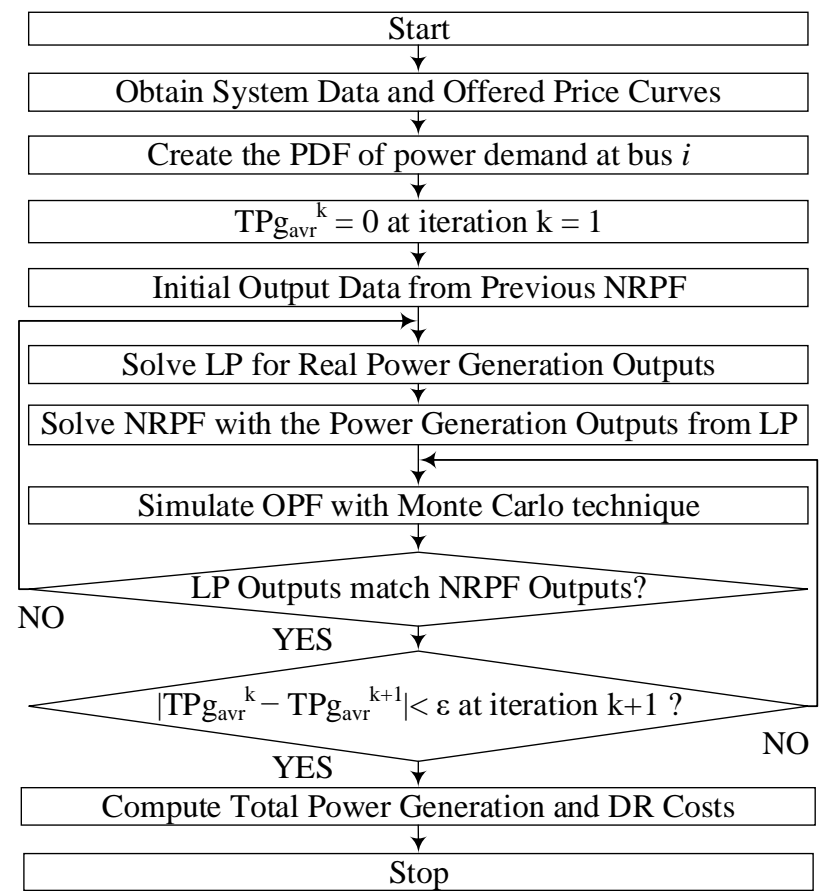

Figure. 3 Framework procedure

the power demand profile is a foremost part of modelling the significance of the random measurement error. When $\sigma_{D}$ becomes wide-ranging, the measurement is moderately imprecise. As the result, a small value of $\sigma_{D}$ will represent a minor error to prove a highly efficient output of random variation.

\subsection{Monte Carlo simulation}

In probabilistic concern, the MCS is commonly used to evaluate the computational model in order to randomize input variations and investigate probabilities outputs. And the framework is illustrated in Fig. 3.

In this paper, the MCS is used for probabilistic power demand simulation and the OPD is run until the average total real power generation of the iteration $k+1\left(T P g_{\text {avg }}{ }^{k+1}\right)$ is close to that of the iteration $k\left(T P g_{a v g}{ }^{k}\right)$. More specifically, the MCS base OPD is run until $\left|T P g_{\text {avg }}{ }^{k}-T P g_{\text {avg }}{ }^{k+1}\right|<\varepsilon$, where $\varepsilon$ is a very small real number. In this paper, the $\varepsilon$ is set to 0.0001 .

MCS is widely used to investigate the power system operation and PDF to forecast the load and uncertainty variables in the system. However, to directly sampling the PDF can lead to infeasible solutions that need further variation process. Therefore, the PTNF could participate in this proposed framework to improve the technique over the existing POPD and lead to better precise results as addressed in Section 5. Without implementing 
PTNF in this study, the simulation will be included a number of infeasible LF solutions during the computational procedure. Therefore, it is noticeably shown that the proposed technique can handle the dispatch solutions considering PRDR effectively and accurately.

\section{Simulation results}

The proposed method is tested with the IEEE 30-bus system [30]. Moreover, the piecewise linear cost function for every generator is provided in
Table 1. The generators' operating costs for each generator are provided to represent its linearized incremental cost curve for each segment of $P_{G i}$ as shown in Section 3.1. Some crucial data for the simulation is provided in Table 2 including $P_{D R i}$ assuming the costs and quantities.

With the piecewise linear staircase cost function, the real power generation of individual segment is dispatched in merit order till reaching the $P_{G i}{ }^{\max }$ maximum real power generation.

Table 1. The generators' operating costs for each generator

\begin{tabular}{|c|c|c|c|c|c|c|c|c|c|c|c|}
\hline \multirow{3}{*}{$\begin{array}{l}\text { Bus } \\
\text { No. }\end{array}$} & \multirow{2}{*}{\multicolumn{2}{|c|}{ Incremental }} & \multirow{3}{*}{\begin{tabular}{|c|c}
$\begin{array}{c}\text { Piecew. Linear } \\
\text { Increm. Cost }\end{array}$ \\
(\$/MWHr)
\end{tabular}} & \multirow{3}{*}{$\begin{array}{l}P G^{\min } \\
(\mathrm{MW})\end{array}$} & \multirow{3}{*}{$\begin{array}{l}P_{G}^{\max } \\
(\mathrm{MW})\end{array}$} & \multirow{3}{*}{$\begin{array}{l}\text { Bus } \\
\text { No. }\end{array}$} & \multirow{2}{*}{\multicolumn{2}{|c|}{ Incremental }} & \multirow{3}{*}{\begin{tabular}{|c|}
$\begin{array}{c}\text { Piecew. Linear } \\
\text { Increm. Cost }\end{array}$ \\
(\$/MWHr $)$ \\
\end{tabular}} & \multirow{3}{*}{$\begin{array}{l}\boldsymbol{P}_{G}^{\min } \\
(\mathrm{MW})\end{array}$} & \multirow{3}{*}{$\frac{P_{G}{ }^{\max }}{(\mathrm{MW})}$} \\
\hline & & & & & & & & & & & \\
\hline & (MW) & (MW) & & & & & (MW) & (MW) & & & \\
\hline \multirow{8}{*}{1} & 50 & 71 & 4.540 & \multirow{8}{*}{50} & \multirow{8}{*}{200} & \multirow{8}{*}{8} & 10 & 25.6 & 4.750 & \multirow{8}{*}{10} & \multirow{8}{*}{135} \\
\hline & 71 & 92 & 5.150 & & & & 25.6 & 41.2 & 5.650 & & \\
\hline & 92 & 110 & 5.600 & & & & 41.2 & 56.85 & 5.870 & & \\
\hline & 110 & 128 & 6.150 & & & & 56.85 & 72.5 & 6.650 & & \\
\hline & 128 & 146 & 6.860 & & & & 72.5 & 88.15 & 7.410 & & \\
\hline & 146 & 164 & 7.150 & & & & 88.15 & 103.8 & 8.150 & & \\
\hline & 164 & 182 & 8.120 & & & & 103.8 & 119.4 & 8.970 & & \\
\hline & 182 & 200 & 8.850 & & & & 119.4 & 135 & 9.350 & & \\
\hline \multirow{8}{*}{2} & 20 & 40 & 5.050 & \multirow{8}{*}{20} & \multirow{8}{*}{180} & \multirow{8}{*}{11} & 10 & 25 & 3.670 & \multirow{8}{*}{10} & \multirow{8}{*}{130} \\
\hline & 40 & 60 & 5.550 & & & & 25 & 40 & 4.350 & & \\
\hline & 60 & 80 & 6.100 & & & & 40 & 55 & 5.670 & & \\
\hline & 80 & 100 & 8.150 & & & & 55 & 70 & 6.050 & & \\
\hline & 100 & 120 & 9.000 & & & & 70 & 85 & 6.670 & & \\
\hline & 120 & 140 & 10.15 & & & & 85 & 100 & 7.170 & & \\
\hline & 140 & 160 & 11.00 & & & & 100 & 115 & 7.970 & & \\
\hline & 160 & 180 & 11.85 & & & & 115 & 130 & 8.950 & & \\
\hline \multirow{8}{*}{5} & 15 & 31.9 & 4.050 & \multirow{8}{*}{15} & \multirow{8}{*}{150} & \multirow{8}{*}{13} & 12 & 28 & 3.100 & \multirow{8}{*}{12} & \multirow{8}{*}{140} \\
\hline & 31.9 & 48.8 & 4.240 & & & & 28 & 44 & 5.350 & & \\
\hline & 48.8 & 65.65 & 4.490 & & & & 44 & 60 & 5.450 & & \\
\hline & 65.65 & 82.5 & 5.150 & & & & 60 & 76 & 6.000 & & \\
\hline & 82.5 & 99.4 & 5.850 & & & & 76 & 92 & 7.600 & & \\
\hline & 99.4 & 116.3 & 6.500 & & & & 92 & 108 & 8.150 & & \\
\hline & 116.3 & 133.15 & 7.200 & & & & 108 & 124 & 9.200 & & \\
\hline & 133.15 & 150 & 8.850 & & & & 124 & 140 & 10.50 & & \\
\hline
\end{tabular}

Table 2. The power demand for the modified 30-bus test system

\begin{tabular}{|c|c|c|c|c|}
\hline $\begin{array}{c}\text { Bus } \\
\text { No. }\end{array}$ & \multicolumn{2}{|c|}{$\begin{array}{c}\text { Power Demand } \\
\text { (MW) }\end{array}$} & \multicolumn{2}{c|}{ PRDR } \\
\hline 2 & $\tilde{P}_{D_{2}}-P_{D R_{2}}$ & 12.70 & 2.604 & 1.422 \\
\hline 5 & $\tilde{P}_{D_{5}}-P_{D R_{5}}$ & 19.00 & 11.30 & 1.062 \\
\hline 7 & $\tilde{P}_{D_{7}}-P_{D R_{7}}$ & 10.90 & 2.736 & 1.062 \\
\hline 8 & $\tilde{P}_{D_{8}}-P_{D R_{8}}$ & 30.00 & 3.600 & 1.122 \\
\hline 12 & $\tilde{P}_{D_{12}}-P_{D R_{12}}$ & 7.500 & 1.344 & 1.260 \\
\hline 21 & $\tilde{P}_{D_{21}}-P_{D R_{21}}$ & 11.20 & 2.100 & 1.122 \\
\hline 30 & $\tilde{P}_{D_{30}}-P_{D R_{30}}$ & 1.900 & 1.272 & 1.122 \\
\hline
\end{tabular}

In this research paper, the reactive power generating cost is not included in the result. In the meantime, the single loading condition is used to test the proposed algorithm.

Based on the LP linear cost function, the limit constraint as mentioned in Eq. (9) is applied to guarantee the well-balanced power generation equivalent to the power demand. Another thing to be taken into this approach is to assign the number of segments, which affects the dispatch solutions from LPOPD. As shown in Table 1, the generators' operating costs for each generator is provided with 8 segments for each cost function to observe very close solutions to the target outputs in verifying with 




Figure. 4 Normal PDF output



Figure. 5 PTNF output within one standard deviation



Figure. 6 PTNF output within two standard deviations

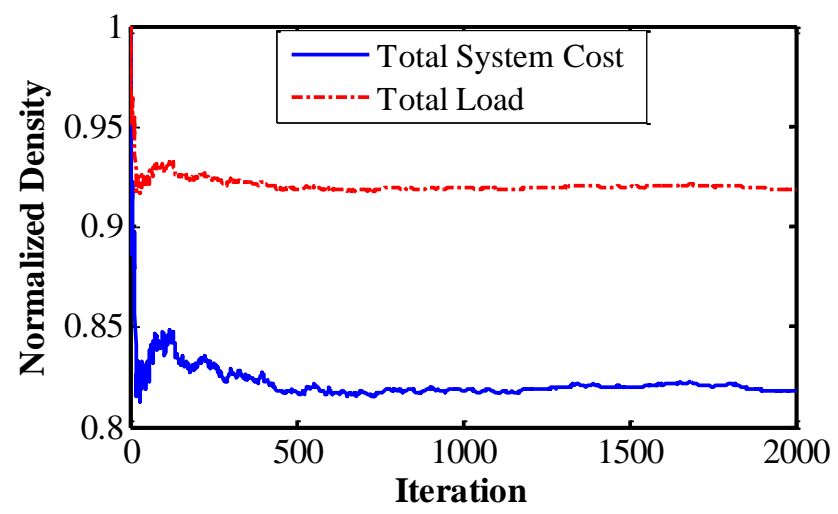

Figure. 7 PTNF output within three standard deviations

the lambda iteration method [21] and the DommelTinney method [30].
Along with each generator data, the power demand data for the modified 30-bus test system are improved to adapt the proposed framework. The PRDRs are regulated on the load bus 2, 5, 7, 8, 12, 21 , and 30 which ranged from 10.6 MW to 94.2 MW assuming to be the dispatchable aggregator loads. In comparing to the fixed price, consumers have participated in the PRDR program by decreasing their energy usage between $11 \%$ to $21 \%$ in the whole year. Evidently, reference [31] is represented by the average $12 \%$ of participants have saved their annual consumption pattern.

\subsection{Investigation of the proposed framework}

The output investigation by the proposed framework with the modified IEEE 30-bus test system as attached are demonstrated to certify the reliability of the results; the simulation 2000 runs were performed by POPD computational procedures as shown in Fig. 3. Figs. 4-7 represented the outputs of total operating cost and total power demand, which are obtained from POPD simulation with and without PTNF. From the simulation output, there is some evidence of how the results have converged.

From the results, normal PDF with the computational framework processed at least 1175 trials to give the convergent solution. Contribution to this study, it was improved after applying the empirical rule mentioned in Section 4. They are involved in the computational procedure by improving to give the convergent solutions at least 495 trials within one standard deviation and at least 713 trials within two standard deviations. In addition, within three standard deviations, the convergence has met at least 975 trials similar to the case of normal PDF. Nevertheless, it was cleared that the standard deviation value $\sigma_{D}$ became widespread when the measurement is moderately inaccurate just like the theorist intended the idea $[5,26]$. It could be converged at least 1175 trials or more with the random numbers from the normal PDF in the framework as shown in Fig. 3 whereas it has the opportunity to get the faster convergent solutions at 495 trials. Meanwhile, the solutions were obtained with slightly errors of mean value between before and after applying the probabilistic model to the system simulation. It is because of the characteristics of simulation methods.

\subsection{Dispatch results}

Concerning the results from Monte Carlo simulation with normal PDF and PTNF, the probabilistic investigation figures are intended that the output from the proposed method is 
demonstrated the convergence significantly. Even though, in the beginning, it seems a little bit worth divergence from the spot solution, it came out after some iterations. It is noticed that the yield is hereby indicated the active power demand, which is functioning to the total operating cost. On the one hand, PRDR will be instanced dependability in this study due to contract in the DR program. In contrast, the reactive power generation and the system losses are not considered in this framework. Still, it is certainly simplified the effectiveness of the proposed context. The dispatch results will be shown in Table 3 which will compare to some relevant methods respectively.

From the experiment results, it showed that the proposed method has satisfied the objective function to the dispatch solutions. To confirm the base case study of OPF, the computational procedure is shown in Fig. 2. It has verified with [30] and it is clarified that the proposed LPOPD is successfully dispatched with a neglected slop error $1.36 \%$ due to the nature of the piecewise linear optimization model. In Table 3 , the POPD is run without PRDR and figured out the total system operating cost dispatch $739.13 \$ / \mathrm{Hr}$. After that, the PRDR is conducted on the system, at the moment, the total system operating cost is dispatched to $601.51 \$ / \mathrm{Hr}$. Although in probabilistic approach and sampling conditions. It continued to carry out the total dispatch operating cost at 445.57 $\$ /$ Hr by using normal PDF random variation input as loading uncertainties at the specified bus as shown in Table 2. Moreover, the rules for PTNF has applied to the LPOPD procedure then the total operating cost becomes much better at $440.45 \$ / \mathrm{Hr}$, $441.24 \$ / \mathrm{Hr}$, and $441.83 \$ / \mathrm{Hr}$ respectively to specific percentage range as mentioned in Section 4.2. To be notified that there must be some clearance payments for PRDR customers about 28.13 \$/MWHr due to the PRDR contracts between customers and $\mathrm{SO}$ in this prospectus.

In addition to Section 5.1, Table 4 indicates the accomplishment of the proposed method comparing to other recent proposed methods. The probabilistic technique and sampling conditions in Section 4 play an important role in the computational procedure by producing only feasible solutions during the simulation. The results are significantly achieved the good performance by applying PTNF in the computational framework. Without PTNF, the feasible load PDF modelling cannot be established efficiently.

Table 3. The dispatch results of the modified IEEE 30-bus test system

\begin{tabular}{|c|c|c|c|c|c|c|c|c|}
\hline \multicolumn{2}{|c|}{ Variable } & \multirow{2}{*}{$\begin{array}{l}\text { Base Case } \\
\text { Load Flow }\end{array}$} & \multirow{2}{*}{$\begin{array}{c}\text { OPD } \\
\text { without DR }\end{array}$} & \multirow{2}{*}{$\begin{array}{c}\text { OPD } \\
\text { with DR }\end{array}$} & \multirow{2}{*}{$\begin{array}{c}\text { POPD } \\
N(\mu, \sigma)\end{array}$} & \multicolumn{3}{|c|}{ POPD with PTNF } \\
\hline & & & & & & $\mu \pm \sigma$ & $\mu \pm 2 \sigma$ & $\mu \pm 3 \sigma$ \\
\hline \multirow{6}{*}{ 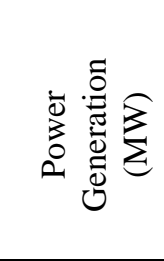 } & \multirow{6}{*}{$\begin{array}{l}P_{G 1} \\
P_{G 2} \\
P_{G 5} \\
P_{G 8} \\
P_{G 11} \\
P_{G 13} \\
\end{array}$} & 55.5 & 79.97 & 71 & 70.19 & 73.64 & 69.74 & 69.34 \\
\hline & & 46.5 & 40 & 48.57 & 24.47 & 20.31 & 23.99 & 24.44 \\
\hline & & 48.3 & 72.88 & 65.65 & 64.55 & 63.46 & 64.79 & 64.68 \\
\hline & & 55.7 & 25.6 & 25.6 & 18.54 & 19.05 & 18.63 & 18.5 \\
\hline & & 35.2 & 40 & 40 & 39.48 & 39.98 & 39.24 & 39.42 \\
\hline & & 45.7 & 28 & 28 & 28.01 & 28 & 28 & 28 \\
\hline \multicolumn{2}{|c|}{$\begin{array}{c}\text { Total Generation } \\
\text { [MW, MVAR] }\end{array}$} & $\begin{array}{c}{[286.71} \\
83.58]\end{array}$ & $\begin{array}{c}{[286.45,} \\
83.6]\end{array}$ & $\begin{array}{c}{[278.82,} \\
82.66]\end{array}$ & $\begin{array}{c}{[245.24} \\
80.5]\end{array}$ & $\begin{array}{c}{[244.44} \\
80.54]\end{array}$ & $\begin{array}{c}{[244.39} \\
80.38]\end{array}$ & $\begin{array}{c}{[244.38,} \\
80.41]\end{array}$ \\
\hline \multicolumn{2}{|c|}{$\begin{array}{l}\text { Total P-Q Load } \\
\text { [MW, MVAR] }\end{array}$} & $\begin{array}{c}{[283.4,} \\
126.2]\end{array}$ & $\begin{array}{c}{[283.16} \\
126.2]\end{array}$ & $\begin{array}{c}{[275.75} \\
126.2]\end{array}$ & $\begin{array}{c}{[242.63} \\
126.2]\end{array}$ & $\begin{array}{c}{[241.84} \\
126.2]\end{array}$ & $\begin{array}{c}{[241.8} \\
126.2]\end{array}$ & $\begin{array}{c}{[241.81,} \\
126.2]\end{array}$ \\
\hline \multicolumn{2}{|c|}{$\begin{array}{c}\text { Total Syst. Losses } \\
\text { [MW, MVAR] }\end{array}$} & $\begin{array}{c}{[3.31} \\
-17.18]\end{array}$ & $\begin{array}{l}{[3.29} \\
-17.17]\end{array}$ & $\begin{array}{l}{[3.08,} \\
-18.11]\end{array}$ & $\begin{array}{c}{[2.6} \\
-20.23]\end{array}$ & $\begin{array}{l}{[2.61} \\
-20.19]\end{array}$ & $\begin{array}{l}{[2.58,} \\
-20.35]\end{array}$ & $\begin{array}{l}{[2.58,} \\
-20.32]\end{array}$ \\
\hline \multicolumn{2}{|c|}{$\begin{array}{c}\text { Total Gen. Cost } \\
(\$ / \mathrm{Hr})\end{array}$} & 791.48 & 739.13 & 573.38 & 417.44 & 412.32 & 413.11 & 413.7 \\
\hline \multicolumn{2}{|c|}{$\begin{array}{l}\text { Total } D R \text { Cost } \\
\text { (\$/MWHr) }\end{array}$} & - & - & 28.13 & 28.13 & 28.13 & 28.13 & 28.13 \\
\hline \multicolumn{2}{|c|}{$\begin{array}{c}\text { Total Syst. Cost } \\
(\$ / \mathrm{Hr})\end{array}$} & 791.48 & 739.13 & 601.51 & 445.57 & 440.45 & 441.24 & 441.83 \\
\hline
\end{tabular}


Table 4. Data comparison with relevant methods

\begin{tabular}{|c|c|c|c|}
\hline $\begin{array}{c}\text { Sampling } \\
\text { Variables }\end{array}$ & $\begin{array}{c}\text { Iter. } \\
{[\text { Trials] }}\end{array}$ & $\begin{array}{c}\text { CPU } \\
{[\mathbf{s}]}\end{array}$ & $\begin{array}{c}\text { Tot. Syst. } \\
\text { Cost }[\mathbf{\$} / \mathbf{H r}]\end{array}$ \\
\hline Normal PDF & 1175 & 33.9 & 445.57 \\
\hline PTNF $(\mu \pm \sigma)$ & 495 & 33.49 & 440.45 \\
\hline PTNF $(\mu \pm 2 \sigma)$ & 713 & 33.56 & 441.24 \\
\hline PTNF $(\mu \pm 3 \sigma)$ & 975 & 33.67 & 441.83 \\
\hline PE $^{[15]}$ & 1504 & - & - \\
\hline JSQN $^{[25]}$ & - & 48.39 & - \\
\hline PEM $^{[18]}$ & - & - & 804.73 \\
\hline
\end{tabular}

PE: Percentiles Estimation

PEM: Point Estimate Method

JSQN: Johnson system and Sobol's quasi-random numbers

\section{Conclusion}

In this paper, the LP based POPD is proposed for solving the power generation dispatch associated with PRDR to minimize total operating cost. The objective function is to diminish the total system cost while compensating between the high peaking cost power generation and PRDR offered. Moreover, the expected short-term load forecast is represented by the normal PDF with PTNF sampling technique. Hence, the proposed method can effectively and efficiently minimize the total power generation cost, while trading off the PRDR cost in the POPD problem with load uncertainty. Consequently, the proposed method enhances the benefits not only the SO but also the consumers, those are able to claim their paybacks by participating in PRDR contracts. The only thing to do is to rearrange the consumptions during the peak periods or time-ahead from SO's request or contract. It is substantiated that the proposed method can potentially be used to deal with the future electricity supply market.

\section{Acknowledgements}

The authors are grateful to Vithedbundit Scholarship at the Suranaree University of Technology for financial support.

\section{References}

[1] A. Chauhan and R.P. Saini, "Size Optimization and Demand Response of a Stand-alone Integrated Renewable Energy System", Energy, Vol.124, pp.59-73, 2017.

[2] F. Babonneau, M. Caramanis, and A. Haurie, "A Linear Programming Model for Power Distribution with Demand Response and Variable Renewable Energy", Applied Energy, Vol.181, pp.83-95, 2016.
[3] F. M. Tuaimah and M. F. Meteb, "A Linear Programming Method Based Optimal Power Flow Problem for Iraqi Extra High Voltage Grid (EHV)", Journal of Engineering, Vol.20, pp.2335, 2014.

[4] W. Ongsakul, S. Chirarattananon, and K. Chayakulkheeree, "Optimal Real Power Dispatching Algorithm for Auction Based Dispatch Problems", In: Proc. of International Conf. On Power Systems, pp.434-440, 2001.

[5] A. J. Wood, B. F. Wollenberg, and G. B. Sheblé, Power Generation, Operation, and Control, Third Edition, John Wiley \& Sons, Inc., Hoboken, New Jersey, 2014.

[6] M. S. Viana, G. M. Junior, and M. E. M. Udaeta, "Analysis of Demand Response and Photovoltaic Distributed Generation as Resources for Power Utility Planning", Applied Energy, Vol.217, pp.456-466, 2018.

[7] C. Li, F. de Bosio, F. Chen, S. K. Chaudhary, J. C. Vasquez, and J. M. Guerrero, "Economic Dispatch for Operating Cost Minimization Under Real-Time Pricing in Droop-Controlled DC Microgrid", IEEE Journal of Emerging and Selected Topics in Power Electronics, Vol.5, No.1, pp.587-595, 2017.

[8] R. Shigenobu, A. Yona, and T. Senjyu, "Optimal Demand Response Considering the Optimal Power Flow in Electricity Market", In: Proc. of International Conf. On Industrial Technology, pp.523-528, 2016.

[9] J. S. Vardakas, N. Zorba, and C. V. Verikoukis, "A Survey on Demand Response Programs in Smart Grids: Pricing Methods and Optimization Algorithms", IEEE Communication Surveys \& Tutorials, Vol.17, No.1, pp.152-178, 2015.

[10] N. G. Paterakis, O. Erdinç, and J. P. S. Catalão, "An Overview of Demand Response: KeyElements and International Experience", Renewable and Sustainable Energy Reviews, Vol.69, pp.871-891, 2017.

[11] T. Müller and D. Möst, "Demand Response Potential: Available when Needed?", Energy Policy, Vol.115, pp.181-198, 2018.

[12] F. A. Rahiman, H. H. Zeineldin, V. Khadkikar, S. W. Kennedy, and V. R. Pandi, "Demand Response Mismatch (DRM): Concept, Impact Analysis, and Solution", IEEE Transactions on Smart Grid, Vol.5, No.4, pp.1734-1743, 2014.

[13] A. Moshari, A. Ebrahimi, and M. FotuhiFiruzabad, "Short-Term Impacts of DR Programs on Reliability of Wind Integrated Power Systems Considering Demand-Side Uncertainties", IEEE Transactions on Power Systems, Vol.31, No.3, pp.2481-2490, 2016. 
[14] R. N. Allan, A. M. Leite da Silva, and R. C. Burchett, "Evaluation Methods and Accuracy in Probabilistic Load Flow Solutions", IEEE Transactions on Power Apparatus and Systems, Vol.PAS-100, No.5, pp.2539-2546, 1981.

[15] K. Chayakulkheeree, "Probabilistic Optimal Power Flow with Weibull Probabilistic Distribution Function of System Loading Using Percentiles Estimation", Electric Power Components and Systems, Vol.41, No.3, pp.252-270, 2013.

[16] S. Zhang, H. Cheng, L. Zhang, M. Bazargan, and L. Yao, "Probabilistic Evaluation of Available Load Supply Capability for Distribution System", IEEE Transactions on Power Systems, Vol.28, No.3, pp.3215-3225, 2013.

[17] J. S. Giraldo-Chavarriaga, J. A. CastrillónLargo, and M. Granada-Echeverri, "Stochastic AC Optimal Power Flow Considering the Probabilistic Behavior of the Wind, Loads and Line Parameters", Ingeniería Investigación y Tecnología, Vol.15, No.4, pp.529-538, 2014.

[18] S. Shargh, B. Khorshid ghazani, B. Mohammadi-ivatloo, $H$. Seyedi, and $M$. Abapour, "Probabilistic Multi-Objective Optimal Power Flow Considering Correlated Wind Power and Load Uncertainties", Renewable Energy, Vol.94, pp.10-21, 2016.

[19] K. Chayakulkheeree, "Probabilistic Load Flow for High Penetration Solar Power Plant Distribution System Using Percentile Estimation of Weibull Probabilistic Distribution Function", In: Proc. of PEA Conf. On Technology for Distribution System Development in the Future, pp.116-121, 2015.

[20] G. Carpinelli, P. Caramia, and P. Varilone, "Multi-Linear Monte Carlo Simulation Method for Probabilistic Load Flow of Distribution Systems with Wind and Photovoltaic Generation Systems", Renewable Energy, Vol.76, pp.283-295, 2015.

[21] D. Villanueva, A. Feijóo, and J. L. Pazos, "Probabilistic Load Flow Considering Correlation between Generation, Loads and Wind Power", Smart Grid and Renewable Energy, Vol.2, pp.12-20, 2011.

[22] B. Matthiss, P. Gaedke, M. Felder, and J. Binder, "Probabilistic Load Flow Methods for Energy Management Schemes in Distribution Grids", In: Proc. of International Conf. On Environment and Electrical Engineering and IEEE Industrial and Commercial Power Systems Europe, pp.1-5, 2017.
[23] B. C. Gu, Z. M. Chen, T. Y. Ji, L. L. Zhang, Q. H. Wu, M. S. Li, and J. H. Huang, "QuasiMonte Carlo Simulation Based Economic Dispatch with Wind Power Integrated", In: Proc. of International Conf. On Innovative Smart Grid Technologies - Asia (ISGT-Asia), pp.264-269, 2016.

[24] P. Jorgensen, J. S. Christensen, and J. O. Tande, "Probabilistic Load Flow Calculation Using Monte Carlo Technique for Distribution Network with Wind Turbines", In: Proc. of International Conf. On Harmonics and Quality of Power, pp.1146-1151, 1998.

[25] L. Zhang, H. Cheng, S. Zhang, P. Zeng, and L. Yao, "Probabilistic Power Flow Calculation Using the Johnson System and Sobol's QuasiRandom Numbers", IET Generation, Transmission \& Distribution, Vol.10, No.12, pp.3050-3059, 2016.

[26] J. Burkardt, The Truncated Normal Distribution, Department of Scientific Computing, Florida State University, 2014.

[27] R. Krenek, J. Cha, and B. R. Cho, "Development of the Convolutions of Truncated Normal Random Variables with Three Different Quality Characteristics in Engineering Applications", Computers and Industrial Engineering, Vol.94, pp.125-137, 2016.

[28] F. Ni, P. H. Nguyen, J. F. G. Cobben, and J. Tang, "Application of Non-Intrusive Polynomial Chaos Expansion in Probabilistic Power Flow with Truncated Random Variables", In: Proc. of International Conf. On Probabilistic Methods Applied to Power Systems, pp.1-7, 2016.

[29] D. Mazzeo, G. Oliveti, and E. Labonia, "Estimation of Wind Speed Probability Density Function Using a Mixture of Two Truncated Normal Distributions", Renewable Energy, Vol.115, pp.1260-1280, 2018.

[30] O. Alsac and B. Stott, "Optimal Load Flow with Steady-State Security", IEEE Transactions on Power Apparatus and Systems, Vol.PAS-93, No.3, pp.745-751, 1974.

[31] J. Wang, M. A. Biviji, and W. M. Wang, "Lessons Learned from Smart Grid Enabled Pricing Programs", In: Proc. of International Conf. On Power and Energy, pp.1-7, 2011. 